\title{
A year in genetics
}

As the year comes to a close and we start to look ahead to 2020, we thought that we would highlight some of our favorite Nature Genetics papers from 2019. This snapshot also captures some of the topics and themes in genetics that we are most excited to see develop in the near future.

W e started off the year by publishing, in the January issue, the first GWAS for attention deficit/hyperactivity disorder (ADHD) to identify genome-wide-significant loci for this important condition. We have seen many interesting and field-advancing studies in neurogenomics this year, among which this GWAS has provided novel insights into both ADHD itself and other related disorders. We hope to see further genetic refinement of complex neuropsychiatric traits in the future.

A pan-cancer analysis on how tumor mutation burden associates with patient survival after immunotherapy treatment was published in the February issue. This comprehensive approach highlights general trends across cancers as well as important differences between cancer types, showing how genomics can inform the immunotherapy field. We are particularly eager to see further advances in this area of using genetics to answer clinically relevant or biologically astute questions about responses to immunomodulatory therapies.

A recent theme in genetics research is the importance of including cohorts of diverse ancestry into large-scale analyses such as GWAS. In the March issue, two studies in the Japanese population, one on type 2 diabetes and one on sequencing the $\mathrm{MHC}$ region, provide novel insights into understanding different diseases, not just for specific populations but in general. We hope to see more studies that analyze diverse global or comparatively under-studied populations and that include trans-ancestry comparisons and relevant results for the broadest swath of the world possible.

How the microbiome influences host health and disease, and how human and microbial genetics interact are areas of considerable interest. The challenge for this complex field is to distinguish causality from mere correlation. In the April issue, a study uses a Mendelian Randomization approach to provide a proof of principle that genetic tools can be utilized to study causal associations in the complex space of hostmicrobiome interactions. Much remains to be explored, so teasing out these causal effects will be a rich area for future genetics studies.

A study in the May issue examines fetalmaternal genetic associations, highlighting the influence of the maternal genome on the fetus and vice versa. Genetics can be used to promote and improve maternal health, and we think that research in this area should be a global priority.

Although we have published a number of plant genome studies this year, including those on the strawberry, pea plant and pineapple, the tomato pan-genome study in the June issue provides a great example of using state-of-the-art genomics approaches to study important questions related to agriculture and plant evolution. We hope to see more agricultural genomics studies that will be biologically interesting while having real-world relevance to critical issues such as crop yield, drought or flood tolerance, and disease resistance.

In the promising area of pharmacogenomics, a study in the July issue provides an example of how GWAS data can be harnessed for drug-target prioritization. This avenue is encouraging for research linking fundamental genetic discovery to clinical applications, and we are eager to see more developments using innovative approaches.

Understanding how the genome functions in three-dimensional space is of high interest in the genetics field. In the August issue, a study analyzing the three-dimensional landscape of Drosophila balancer chromosomes provides insights into the relationship between chromatin organization and transcription. So much still remains unknown about the regulation, dynamics and consequences of genome interactions, and we look forward to seeing this exciting field develop further.
At the nexus of single-cell and CRISPR screen technologies is a study, in the September issue, using a pooled single-cell CRISPR-Cas9 screen to identify factors associated with epithelial-to-mesenchymal transition. We hope to see innovative applications of new single-cell-analysis approaches, as well as creative ways of harnessing CRISPR technologies to answer genetics or genomics questions.

By uncovering synergistic effects of common schizophrenia variants, a study in the October issue underscores the need to incorporate information about genetic background when studying disease effects. We hope to see studies that utilize genetic-interaction data as well as those that consider the genome as a whole, characterizing polygenicity and building comprehensive risk scores.

Much information is known about the parts of the genome that are straightforward to sequence and analyze. Complex regions, such as short tandem repeats, are far less well characterized. The November issue includes a study examining the effects of short-tandem-repeat variation on gene expression and how these variants associate with diseases or complex traits. More extensive analysis of not only repeat regions but also other types of variations, such as indels or large structural variants, will provide greater insights into the different genetic contributions to disease.

Finally, in the December issue, a comprehensive study on the mutational footprints of cancer therapies provides a molecular window into the genomic consequences of widely used treatments. Understanding the effects of chemotherapies on the genome and how resistance develops should lead to safer and more effective treatment regimens for patients with cancer, and we encourage more work in this area. $\square$

Published online: 29 November 2019 https://doi.org/10.1038/s41588-019-0553-1 